\title{
Welcome to the Atomic Weapons Establishment
}

\author{
With the launch of a powerful laser facility, Britain's most secretive \\ lab is opening up to academics. Geoff Brumfiel secures a preview.
}

$\Lambda$

ldermaston is a picturesque English village with red-brick houses, tidy gardens and an inviting local pub. But just to the south lies a much more forbidding set of buildings. Behind double-fencing and guarded gates is the sprawling campus of the Atomic Weapons Establishment (AWE), Britain's most hush-hush research laboratory. The 304-hectare facility, a converted Second World War airfield, is home to the roughly 4,000 scientists, engineers and technicians who are responsible for maintaining Britain's nuclear warheads.

The Ministry of Defence, which oversees the AWE, is notoriously tight-lipped about the lab's activities, and scientists who work there generally try to keep a low profile. But within the next few months, the AWE's most ambitious and expensive scientific project is due to be completed, and it is prompting the lab to open up its doors - at least a chink.

The new Orion facility will be home to 12 high-powered laser beams capable of heating and compressing material to millions of degrees Celsius in less than a nanosecond. Housed in a gleaming building the size of a soccer pitch, the laser system will provide physicists at Aldermaston with crucial data about how components of their ageing nuclear weapons behave. Under current plans, around $15 \%$ of Orion's time will be offered to academics wanting to study conditions on stars or inside giant planets. And in that open spirit, researchers there invited a Nature reporter in for a look around.

In most respects, Orion is the smaller cousin of the US National Ignition Facility (NIF) in Livermore, California, which is already running academic experiments. When operating at full steam, the NIF will use 192 lasers to create around 4 million joules of energy, some 100 times more powerful than Orion. What makes the AWE's laser notable is the exquisite precision that it will give researchers in controlling the heat and compression exerted on the materials placed in its target chamber - and the fact that the AWE is sharing it at all.

The motivations for collaboration are not entirely selfless. The defence establishment wants to provide scientists inside the security cordon with the sort of intellectual stimulation needed to keep them on their toes; it also has an incentive to nurture the wider community of physicists from which it draws its staff. "We want to demonstrate that we maintain high standards for our science," says Daryl Landeg, the AWE's chief scientist. And academics far beyond Aldermaston are keen to cross the fence. At present, Europe has only a handful of comparable laser facilities, says François Amiranoff, director of the Laboratory for the Use of Intense Lasers at the École Polytechnique near Paris. "A facility like Orion is very, very interesting for the scientific community," he says.

\section{Secrecy rules}

Britain established its atomic-weapons programme at the current AWE site in 1950, after close involvement in the Manhattan Project. The campus, which also houses facilities for manufacturing and storing sensitive nuclear materials, is shielded by the nation's strict secrecy laws, and has historically shunned visitors. Things

have started to change over the past decade, says Steven Rose, a physicist at Imperial College London who headed the AWE's plasmaphysics division from 2001 to 2004. "The old argument that secrecy was paramount I think perhaps holds less sway these days," he says. "They've realized that you can do some things in collaboration with the outside world."

Enter Orion, the $£ 183$-million (US\$274million) laser on which construction began in

"The defence ministry is notoriously tightlipped about the lab's activities." 2005. The machine has ten conventional lasers (see graphic), each of which can deliver 500 joules of energy in about a nanosecond (a billionth of a second). It also has two short-pulse lasers, which deliver the same amount of energy in just half a picosecond (a trillionth of a second).

Inside Orion's main building, workers in Teflon suits and hairnets are busily scrambling around gigantic white scaffolding. The structure will soon hold the mirrors, amplifiers and lenses needed to boost and focus the 12 beams onto their target, which lies in a separate chamber behind 1.5 metres of solid concrete, a shield necessary to contain the radiation generated when the laser beams hit. The exceptional cleanliness in the laser halls and target area (and expected of visitors) is about more than aesthetics: a stray hair in the path of the intense beam could cause irregularities and crack an expensive mirror or grating.

Orion's main mission, like that of

ORION LASER

Ten long-pulse and two short-pulse lasers allow precise control of temperature and pressure in target materials.

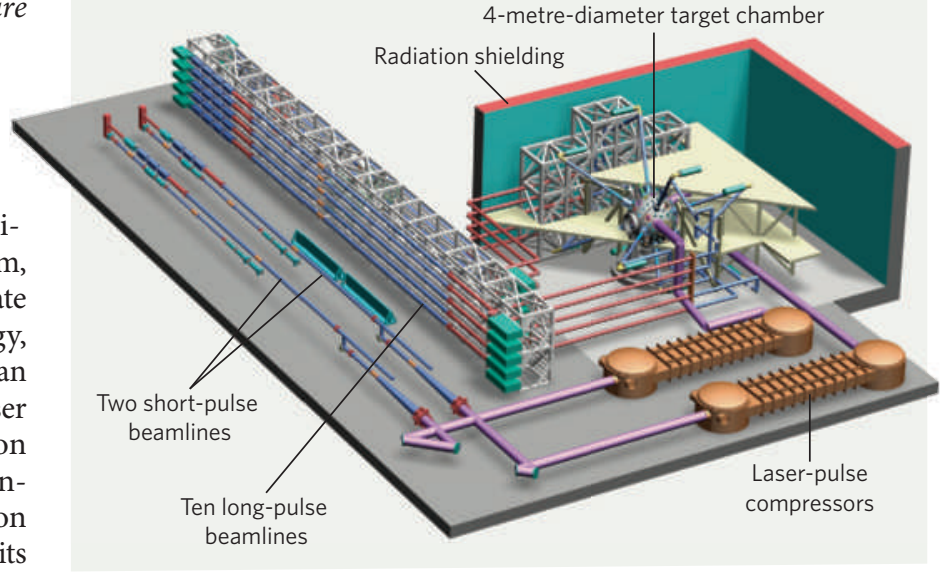
the NIF, is to explore how nuclear weapons work, particularly as they get older. In 1998, Britain ratified the Comprehensive Nuclear-TestBan Treaty, an international agreement prohibiting tests of nuclear weapons. Scientists in Britain and worldwide have therefore been busy developing computer models to simulate nuclear warheads and work out whether the weapons will still detonate after decades in storage, and what type of detonation will result. What is missing, however, are actual data.

US scientists hope that the more 


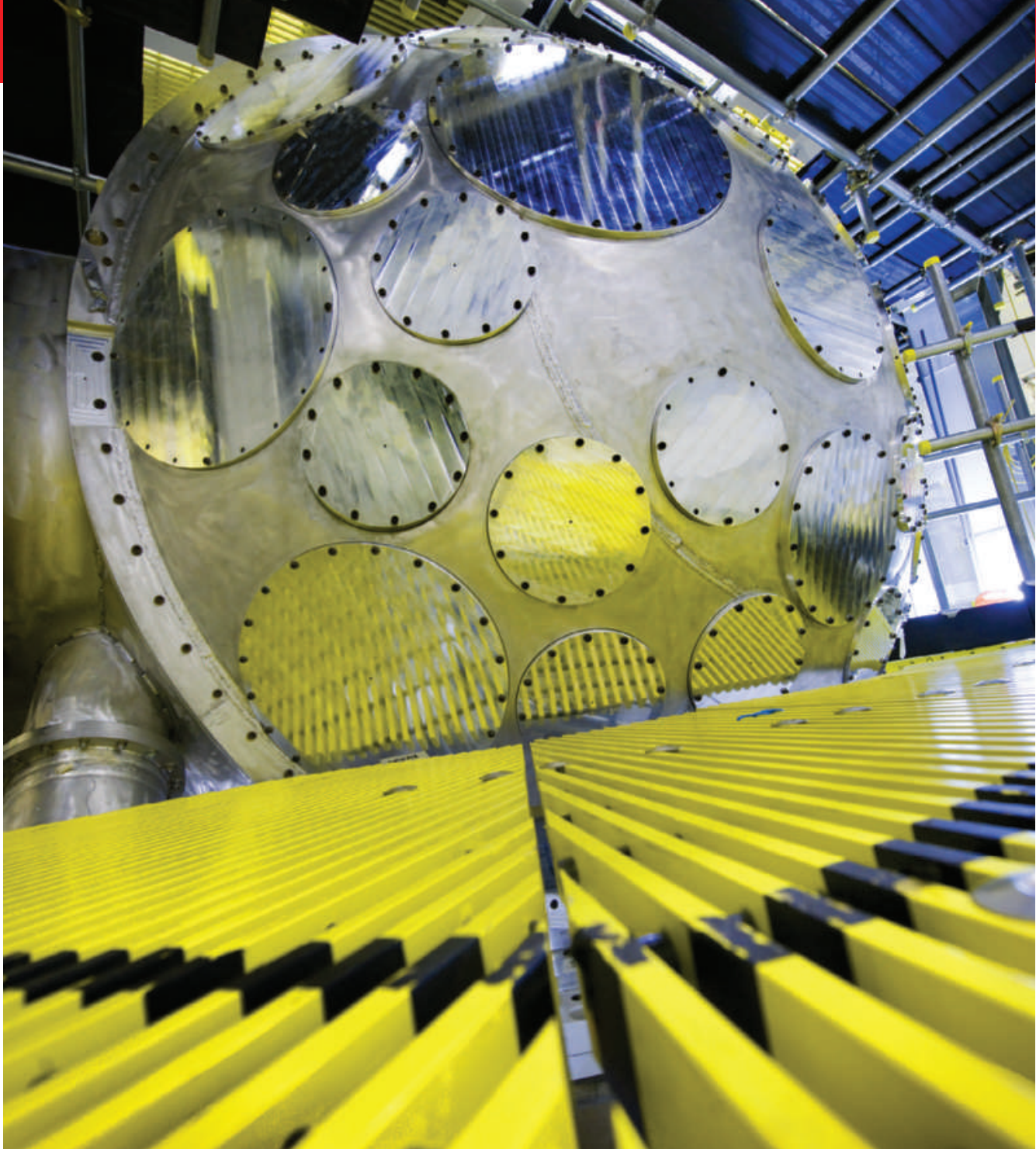

Target chamber: Orion's twelve lasers will explore conditions inside ageing thermonuclear weapons.

powerful NIF will contribute some of those data, by generating temperatures and pressures so high that they will spark nuclear fusion in small quantities of two hydrogen isotopes, deuterium and tritium. This fusion process would resemble conditions inside the most powerful stage of a modern thermonuclear weapon.

If the NIF is a thermonuclear hammer, then Orion is a scalpel. The smaller facility will never achieve full-scale fusion, but it will be able to carefully control conditions inside test materials such as uranium. Pressure and temperature usually go hand-in-hand, explains Peter Roberts, head of the AWE's plasma-physics department. "You pump up a tyre with a bicycle pump and it gets hot," he says. But Orion can get around this. It can compress a material with its long, nanosecond pulses then suddenly heat it with its very short, half-picosecond pulses. The result is 'isochoric heating, an unusual condition in which a material is heated so quickly that it doesn't have time to expand. This capability allows Orion to probe materials at wide-ranging combinations of temperatures and pressures.

In particular, researchers will use Orion to explore two key parameters for materials used in nuclear weapons: their opacity and their equation of state. The first describes how radiation travels through a material — in this case, the two stages that make up a weapon. The first stage, or primary, is a few kilograms of plutonium that are compressed by conventional explosives until they begin a runaway nuclear reaction. The radiation from that reaction is then focused onto the 'secondary', the stage in which hydrogen isotopes create a much larger blast using nuclear fusion. Researchers want to know what the opacity is and how it changes with age so that they can model radiation's flow from the primary to the secondary and verify whether the warheads will still work. The other parameter - the equation of state - describes how a material behaves at enormous pressures and temperatures. By generating data on these and other crucial parameters, Orion will give nuclear-weapons scientists the information they need to ensure that their models are correct. "You can't look this stuff up," Rose says.

The researchers running the NIF often emphasize the giant laser's applications in energy production and fundamental science over its military role; it could, for example, lead to new reactors that produce electricity using tiny fusion implosions. Orion's scientists are much less circumspect. "We're working on weapons physics fundamentally," Roberts says. Nevertheless, he and others at the lab are eager to give civilian scientists an opportunity to use the laser - and the academics are eager to try out its capabilities. "It will have significant characteristics that no other laser in the United Kingdom or indeed Europe has," says Mike Dunne, director of the Central Laser Facility at the Rutherford Appleton Laboratory near Didcot, UK. Dunne says that Orion should relieve the strain on the facility's Vulcan laser, Orion's massively oversubscribed civilian counterpart.

\section{Extreme conditions}

Physicists studying astronomical objects find themselves in a situation not dissimilar to that of nuclear-weapons scientists: unable to recreate the extreme conditions inside a star, for example, and largely dependent on complex computer models to show how they work. Orion will not reach stellar temperatures and pressures, but it will be able to create flows of hot ionized gas with the sorts of high magnetic fields and temperatures that mimic parts of stars and other astronomical objects. By scaling up the data from Orion, astrophysicists should be able to improve their models, Dunne says.

The laser facility can also inform more abstruse, fundamental work in atomic physics. Current theory does well at describing normal matter and extremely hot matter that has been stripped of electrons. But it cannot describe situations in which atoms are subjected to high levels of radiation without losing all their electrons. By heating a test material, then probing it with its short-pulse beams, Orion can provide data that can extend existing theories into this middle region, says Amiranoff. This warm, dense matter may exist at the heart of gas giants such as Jupiter, or even within Earth's core.

Scientific collaborations are not slated to begin on Orion until the second half of 2012, and proposals to use the facility will be submitted through the system being used for Vulcan and other UK lasers, rather than being determined by weapons scientists. This open peer-review of proposals "is absolutely critical to gaining the confidence of the community", Dunne says. "If some committee inside of the wire assessed relative merits, there would always be the suspicion that they were picking topics that helped their programme rather than just because they were good science."

Back on the AWE's campus, the weapons scientists are eagerly preparing for their new guests. Tom Bett, who helps to manage construction, shows off a data-analysis room purpose-built for unclassified visitors and lined with sleek computer terminals. (The weapons work will be done in a separate room, he explains, and the data will be stored on servers locked inside vaults.)

On the ground level, floor-to-ceiling windows illuminate a bright reception area the first thing that visiting researchers will see as they are welcomed to the new laser facility. "Those windows," Bett adds proudly, "are all bullet-proof."

Geoff Brumfiel is a senior reporter for Nature based in London. 